\title{
Uncoupling memory traces
}

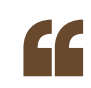 \\ it is possible to uncouple the context of a memory from its valence \\ ce} its valence
The way we feel about a past experience can change over time while other details of the memory remain intact. The cellular mechanisms underlying this phenomenon are not well understood, although we know that the emotional aspects and the spatial contexts of memories are encoded by the amygdala and the hippocampus, respectively. In a new optogenetics-based study, Tonegawa and colleagues artificially altered the emotional valence of a memory in mice and show that this probably involves a change in the connectivity between hippocampal and amygdalar memory traces.

The authors used a viral vector to deliver a channelrhodopsin 2 (ChR2)-mCherry fusion protein to neurons in the hippocampal dentate gyrus (DG) or in the basolateral complex of the amygdala (BLA) of

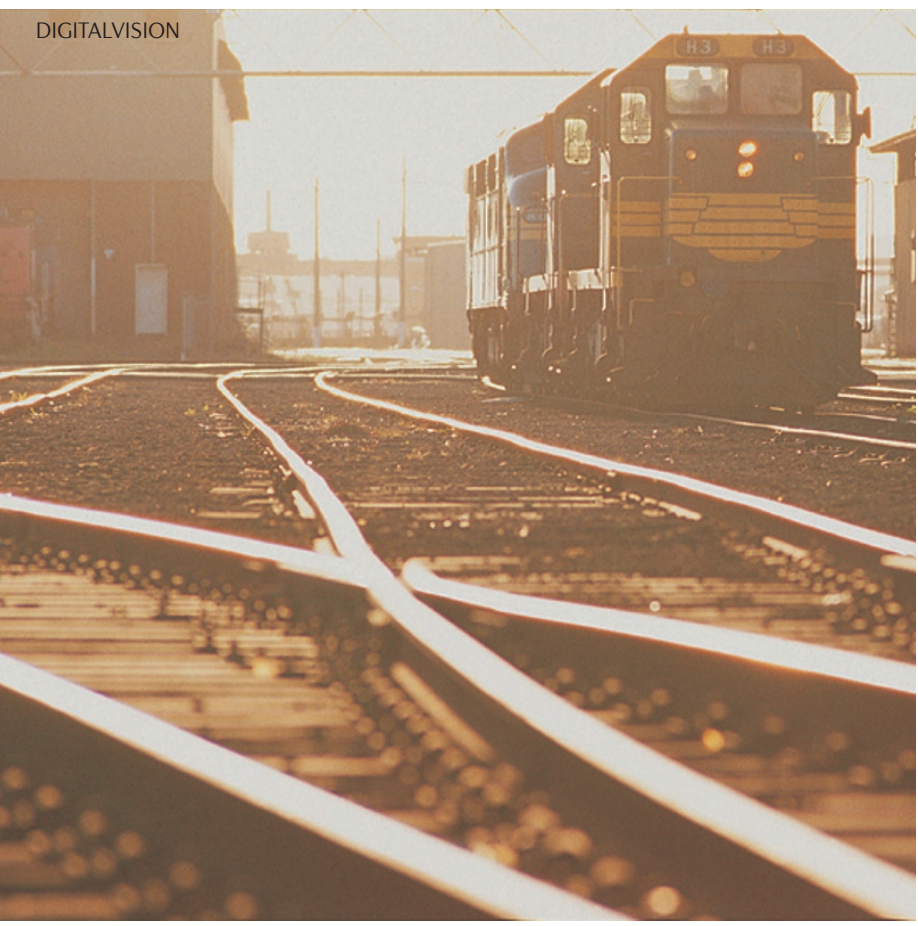

mice. ChR2 expression was under control of both the $c$-fos promoter (to restrict it to activated neurons) and a tetracyclin response element (so that expression could be prevented by giving the animals doxycyclin (Dox)).

After Dox was removed from the diet, the animals underwent either contextual fear conditioning or reward conditioning. In both groups, the procedure resulted in the creation of a memory trace (that is, an 'engram') of activated, ChR2expressing, mCherry-labelled neurons in both the DG and the BLA. The mice were then put back on Dox to prevent further ChR2 expression.

Two days later, the mice had to explore a new environment. When a fear-conditioned mouse was in a particular 'target' area of the enclosure, the authors light-activated the ChR2expressing cells in the DG or BLA that encoded the original fear memory. Reactivation of either fear engram caused the mice to avoid this target area. Conversely, when the authors reactivated the engram in the DG or BLA of reward-conditioned mice, the mice spent more time in the target area than the control mice.

But which engram drives these behavioural responses - the DG engram or the BLA engram? To investigate this question, the authors exposed fear-conditioned mice to a rewarding stimulus in their home cage while light-stimulating the fearmemory traces in the DG or the BLA. Two days later, the authors again reactivated the memory traces while the mice were in the target area of a new context. Activation of the DG engram now induced a preference for the target area, whereas stimulation of the BLA engram did not. Similarly, the authors could change the valence associated with the DG engram - but not the BLA engram - in reward-conditioned mice by light-stimulating the DG memory trace and simultaneously exposing the mice to footshocks.

Together, these findings suggest that both DG and BLA engrams can drive a behavioural response but that the valence associated with a memory engram in the DG can change, whereas the valence of a BLA engram remains associated with the original experience. This indicates that it is possible to uncouple the context of a memory from its valence and suggests that BLA neurons encode a particular valence (either fear or reward) that cannot be changed.

The authors further showed that light-stimulation of a DG engram resulted in activation of mCherrylabelled BLA cells, suggesting that the two engrams are functionally connected. Importantly, the proportion of mCherry-labelled BLA cells that were activated by light-stimulating the DG engram was greatly reduced following reversal of the valence associated with the DG engram. This suggests that changing the valence of an existing memory probably involves the DG cells connecting with a different subset of BLA neurons - presumably a subset that encodes the new valence.

With the methodological tour de force applied in this study, the authors have provided insights into the mechanisms by which good memories can turn bad - and vice versa.

Leonie Welberg

ORIGINAL RESEARCH PAPER

Redondo, R. L. et al. Bidirectional switch of the valence associated with a hippocampal contextual memory engram. Nature http://dx.doi. org/10.1038/nature13725 (2014) 